\title{
Therapeutic Innovations for Targeting Childhood Neuroblastoma: Implications of the Neurokinin-1 Receptor System
}

\author{
MICHAEL BERGER and DIETRICH VON SCHWEINITZ \\ Department of Pediatric Surgery, Research Laboratories, \\ Dr. von Hauner Children's Hospital, Ludwig-Maximilians-University, Munich, Germany
}

\begin{abstract}
Neuroblastoma is the most common solid extracranial malignant tumor in children. Despite recent advances in the treatment of this heterogenous tumor with surgery and chemotherapy, the prognosis in advanced stages remains poor. Interestingly, neuroblastoma is one of the few solid tumors, to date, in which an effect for targeted immunotherapy has been proven in controlled clinical trials, giving hope for further advances in the treatment of this and other tumors by targeted therapy. A large array of novel therapeutic options for targeted therapy of neuroblastoma is on the horizon. To this repertoire, the neurokinin-1 receptor $(N K 1 R)$ system was recently added. The present article explores the most recent developments in targeting neuroblastoma cells via the NKIR and how this new knowledge could be helpful to create new anticancer therapies agains neuroblastoma and other cancers.
\end{abstract}

Neuroblastoma is the most common solid extracranial malignant tumor in children and the most common in infants. In the United States, there are roughly 700 new cases of neuroblastoma diagnosed each year, which accounts for 1 case in every 100,000 children. Depending on which literature to cite, neuroblastoma accounts for approximately $8 \%$ of all malignant childhood tumors and is responsible for

This article is freely accessible online.

Correspondence to: Michael Berger, Department of Pediatric Surgery, Dr. von Hauner Children's Hospital, Ludwig-Maximilians-University, Lindwurmstrasse 4, 80337 Munich, Germany. Tel: +49 89440057811, Fax: +49 89440057815, e-mail: michael.fabian.berger@gmail.com

Key Words: Neuroblastoma, neurokinin-1 receptor, substance $\mathrm{P}$, targeted therapy, pediatric cancer, review. up to $15 \%$ of cancer related deaths in children (1). Neuroblastoma is one of few solid tumors and the only childhood solid tumor in which an effect of targeted immunotherapy has been proven by large, randomized controlled clinical trials. In addition to this success, the field is currently exploring a multitude of innovative and potentially powerful anticancer targets, including the neurokinin-1 receptor (NK1R) system. In this article, we seek to explore the recent advances in targeting neuroblastoma cells specifically via this novel approach as well as the potential implications for the successful treatment of this and other tumors.

\section{Molecular Characteristics of Neuroblastoma}

Neuroblastoma is an extremily heterogenous tumor, both from a clinical and a biological perspective. For example, while some tumors show very aggressive behavior and have a dismal prognosis, others regress spontaneously. Much effort has been invested over the last decades to identify biological and molecular markers that correlate with the clinical behavior and can therefore predict outcome and guide an optimal therapeutic approach. As a matter of fact, due to the success the field has seen in clarifying important biological characteristics and molecular patterns in neuroblastoma, this tumor often functions as a paradigm for biological risk assessment and treatment assignment (2).

Perhaps the most important biological characteristic that predicts clinical behavior in neuroblastoma is the amplification of the $M Y C N$ proto-oncogene in the genome of neuroblastoma cells. The $M Y C N$ oncogene lies within a region of the distal short arm of chromosome $2(2 \mathrm{p} 24)$ and is overexpressed in about $25 \%$ of all neuroblastomas (defined as greater than 10 copies of the gene per cell). Interestingly, about $40 \%$ of neuroblastomas with advanced 
stages carry MYCN amplification, while only $5-10 \%$ of patients with low stage disease show it. This finding is in accordance with the well-known fact that amplification of the MYCN gene correlates heavily with rapid tumor progression and poor outcome. As a matter of fact, in the current Children's Oncology Group (COG) Neuroblastoma risk stratification, unless the tumor is INSS stage I all tumors that show MCYN gene amplification are considered high-risk tumors and are treated accordingly with intensified chemotherapy following myeloablative therapy.

Another important biological characteristic of neuroblastoma is its DNA content. A normal diploid cell has 46 chromosomes, representing 2 copies of each of the 23 chromosomes. In neuroblastoma, this number can be altered quite considerably, and oftentimes many more chromosomes can be found. For example, it is believed that more than half of neuroblastomas are triploid or near-triploid, containing the according number of chormosomes (58 and 80, respectively). The remainders of tumors are considered to be either near-diploid (35-37 chromosomes) or near-tetraploid (81-103 chromosomes) (3). According to their ploidy, one can calculate a DNA index by using the ratio of the actual number of chromosomes to the expected number of 46 (in a normal diploid cell). The DNA index, by representing the DNA content, was found to correlate with outcome and survival rates, although to a much lesser degree than what has been described for the $M Y C N$ gene amplification. At this time, DNA content only has minimal impact on risk stratification within the current COG staging system and potentially influences risk stratification only for infants aged 12-18 months with metastatic disease and infants with $4 \mathrm{~S}$ disease.

Allelic deletions on chromosomes $1 \mathrm{p}$ and $11 \mathrm{q}$ have, similar to amplification of the $M Y C N$ gene and the tumor cell's DNA content, been identified as independent prognostic markers in neuroblastoma. Allelic deletions on chromosomes 1p have been described in about $30 \%$ and deletions in 11q in about $40 \%$ of tumors. For deletion of $1 p$, a loss of a tumor surpressor gene called CHD5 in the region of $1 \mathrm{p} 36.31$ is believed to be the culprit of this altered clinical behavior. Interestingly, deletion of $11 \mathrm{q}$ has been found to be inversely related to amplification of $M Y C N$, yet despite this inverse correlation by itself is a marker for worse prognosis. In the current COG protocol, the intensity of the therapy is altered for intermediate risk neuroblastomas depending on the allelic status regarding $1 \mathrm{p}$ and $11 \mathrm{q}$.

\section{Risk Stratification and Conventional Treatment Strategies}

Neuroblastoma is a very heterogenous tumor, ranging from spontaneous and complete regression to an aggressive, therapy-resistant phenotype with a dismal prognosis. Accordingly, current treatment strategies for neuroblastoma account for this diversity by risk assessment and treatment assignment based on a large range of clinical, histological, molecular and biological markers, the latter two crystalizing themselves as the most important. Current risk stratification in neuroblastoma has recently been reviewed extensively, and as mentioned above, it now serves as a paradigm for risk assessment based on biological characteristics of the tumor and has been described extensively elsewhere (2). Therefore, we here summarize only the key points relevant to the theme of this article.

The most important clinical variables (such that appear to have independent prognostic value) in neuroblastoma for children are age and stage at diagnosis. For example, the younger a child is at diagnosis, the better its prognosis, independent of stage. This correlation appears to continue over time and, depending on the literature to cite, there appears to be an age cut-off of 460 days (London et al. (4)) or 18 months (Moroz et al. (5)). Current treatment protocols of the COG use this insight as part of their risk stratification. For example, patients of ages 12 to 18 months with stage 3 disease are now considered intermediate instead of high risk as long as the tumor is not MYCN amplified (but may have unfavorable histology). Even for stage 4 disease, as long as the tumor has all favorable biological features, in this age group the risk will be considered as intermediate, meaning these children will receive less intensive chemotherapy compared to the high-risk protocol.

Stage individually, like age, seems to be an independent prognostic factor in neuroblastoma. The current staging systems include the International Neuroblastoma Staging System (INSS) as well as the newer neuroblastoma staging system formulated by Monclair et al. in 2009 on behalf of the International Neuroblastoma Risk Group (INRG) (6). The INSS is a surgicopathological staging system while the INRG system focuses on radiographic imaging. While the INSS is largely used throughout the world, it depends heavily on the aggressiveness of the surgeon, as well as his precision in sampling the correct lymph nodes. Further, it cannot adequately stage those tumors that are being observed (instead of being biopsied or resected). The INRG tried to address these shortcomings by developing a complex staging system on the basis of the absence (L1) or presence (L2) of 20 imagedefined risk factors associated to different anatomical relations of the tumor. The staging system in its essence boils down to whether or not vital structures (for example, major vessels) on imaging appear to be encapsuled by tumor. Although somewhat cumbersome to apply, the abscense of imagedefined risk factors has been shown to correlate well with safe and complete tumor resection and is less dependent on the aggressiveness of the individual surgeon (6).

One critical feature of neuroblastoma is its histopathology. Neuroblastomas are divided into four histologic types graded by the amount of surrounding Schwannian stroma. Essentially, 
the more Schwannian stroma is present, the more mature the tumor is believed to be. The four subtypes - ganglioneuroma (Schwannian dominant), ganglioneuroblastoma-nodular (composite, Schwannian stroma-rich/stroma dominant and stroma poor), ganglioneuroblastoma-intermixed (Schwannian stroma-rich), and neuroblastoma (Schwannian stroma poor), are further differentiated into favorable or unfavorable histology. The later distinction is made based on the nuclear morphology of the neuroblastic cells (mitosis-karyorrhexis index (MKI)) as well as the age of the child $(2,7)$.

The most important molecular and biological aberrations in neuroblastoma are, as mentioned above in greater detail, DNA content (ploidy) and $M Y C N$ status. As a matter of fact, the correlation between $M Y C N$ amplification and advanced disease, tumor progression, and poor prognosis is so strong that any tumor that carries it is considered high risk, independent of stage, age, histology or other biological markers. The only exception to this is a stage I tumor, defined as a localized tumor with complete resection and microscopically negative ipsilateral lymph nodes, which is considered low risk despite $M Y C N$ amplification $(8,9)$. The DNA content is not as strong a predictor of outcome as $M Y C N$ amplification. In the current COG risk stratification protocol, DNA content potentially impacts the risk assignment only of infants between 12 and 18 months of age with metastatic disease or of infants with $4 \mathrm{~S}$ disease (2).

\section{Therapeutic Innovations in Neuroblastoma}

The large repertoir of potential targets in neuroblastoma was recently and excellently reviewed by Esposito et al. (10). Therefore, we will only briefly summarize the most important current developments and then focus in more detail on targeting the neurokinin-1 receptor.

Current therapy of childhood neuroblastoma follows specific risk stratification and ranges from surgery alone with observation in certain tumors considered low risk to intensive induction chemotherapy, myeloablative consolidation therapy with stem cell rescue, and targeted therapy for minimal residual disease. The later insight is particularly important for the topic of this article. At this time, neuroblastoma is the only solid childhood tumor in which targeted therapy is part of a standard treatment regimen. By using specific antibodies against GD2, new therapies can now potentially target neuroblastoma cells selectively and mark them for recognition by immune cells. GD2 is a sugar-fat molecule that is expressed on the surface of neuroblastoma cells. Because this marking of GD2 involves an antibody (the antibody against GD2), the immune system can now recognize this cell as foreign or dangerous and will eliminate the cell that carries it. This combination of targeted therapy and immunotherapy is what is ultimately called targeted immunotherapy.
The FDA recently approved the first drug of this kind for the use in high-risk patients with neuroblastoma. Unituxin (dinutuximab) showed considerably good results in recent clinical trials leading up to the approval by the FDA. In a phase II clinical trial, $40 \%$ of children with chemotherapy resistant neuroblastoma who received the mouse monoclonal anti-GD2 antibody 3F8 showed a tumor response (11). The antibody that was used was altered to decrease the immunogenicity of the murine antibody by constructing it as a chimeric antibody in which the variable regions of murine antibody IgG3 antiganglioside GD2 antibody 14.18 were combined with the constant regions of human IgG1-k.

In a phase III clinical trial, 226 children with high-risk neuroblastoma were evaluated. In all of these children, the tumors shrank or disappeared after treatment with multipledrug chemotherapy and surgery, followed by additional intensive chemotherapy and subsequent bone marrow transplantation and radiation therapy. After all of these procedures, all participants received randomly assigned either an oral retinoid drug, isotretinoin, or Unituxin, in combination with additional molecules that stimulate the immune system, such as interleukin-2 and granulocytemacrophage colony-stimulating factor. These stimulating hormone-like molecules appear to enhance the activity of Unituxin and isotretinoin and are usually given along with these drugs (12). After two years, in the mentioned study 66 percent of children who received the Unituxin combination were alive and free of tumor growth or recurrence, compared to only 46 percent of the children in the control group (who were treated with isotretinoin alone, which was the former standard of care). After a data update at a later time point, 73 percent of those who received the Unituxin combination were alive, compared with only 58 percent of those receiving isotretinoin alone (the NNT for Unituxin was 7). Consequently, despite a few significant side effects including irritation of nerve cells, severe pain, and upper airway swelling with difficulty breathing, low blood pressure, bone marrow suppression, and a few others, targeted immunotherapy with Unituxin is currently standard for all high-risk neuroblastoma patients. The approval of Unituxin by the FDA was the first approved new cancer drug for children in over twenty years.

Immunotherapy is powerful and has tremendous potential. Unfortunately, despite the success in the treatment of a few adult tumors and in neuroblastoma, substantial challenges with targeted therapy, including targeted immunotherapy, remain. Nobody yet knows whether tumor cells can escape targeted therapy or even targeted immunotherapy the way they escape the body's immune system and chemotherapy. The hope is that they cannot, but many researchers in the field worry that with repeated exposure - similar to the effects seen for chemotherapy - the cancer cells may find way to escape our immune system. In this sense (but not 
necessarily for this reason), AstraZeneca's pin up drug for targeted therapy Imfinzi in a global, multicenter Phase III trial recently failed to improve the progression-free survival (PFS) in previously-untreated patients with metastatic (Stage IV) 1st-line non-small cell lung cancer (NSCLC). Imfinzi's active component is durvalumab, a human monoclonal antibody directed against programed death ligand-1 (PD-L1), which blocks PD-L1 interaction with PD-1 and CD80 on T cells and thereby counters a tumour's immune-escaping tactic and induces a favorable immune response (a summary of these preliminary results of the MYSTIC trial can be found on AstraZeneca's website).

One problem with targeted therapies that has emerged already is that they are often only cytostatic, whereas standard chemotherapy agents are cytotoxic. Therefore, more often than not, the few targeted therapies that do show a clinically proven effect have to be used together with chemotherapy. As in advanced neuroblastoma, this therapy does not yet replace but is administered in addition to the intensive and debilitating chemotherapy, surgery, and bone marrow transplantation. Thus, if targeted therapy cannot replace or reduce the necessity of chemotherapy, it loses its magic.

In this respect, targeting the ALK thyrosine kinase has important implications for targeting neuroblastoma. Mutations in $A L K$ are known to play a pivotal role in the development of neuroblastoma and such mutations are found specifically in familial cases of neuroblastoma. Mutations in $A L K$ seem to play a role not only in neuroblastoma, but also in a large variety of other malignancies. Thus, targeting $A L K$ is currently considered a promising target in many cancers.

With respect to neuroblastoma, it was found that ALK serves as a target of the MYCN transcription factor. Consequently, one must automatically link the implications of $M Y C N$ amplification on prognosis to ALK, making it an even more unique target in neuroblastoma. Originally, targeting of ALK was tried with antibodies, but was not found very successful. Newer generations of ALK inhibitors intrinsically inhibit this receptor system. The most known anti-ALK drug is the compound crizotinib and is currently marketed by Pfizer. It has shown promising results in the treatment of patients with deregulated ALK function (13-15). A large variety of additional molecular targets exist for neuroblastoma and are currently being investigated as anticancer agents $(2,10)$. To date, none other to what was mentioned above has made it to the bedside.

The NK1R system in cancer. The existence of the neurokinin1 receptor and its ligand are known for decades, however, its involvement in the formation of cancer is a rather recent understanding. The gene for this receptor, TACR 1 , is located on chromosome 2 in humans and consists of a total of 5 exons. The full-length version of the NK1R consists of a total of 417 amino acids. The NK1R belongs to a larger family of neurokinin receptors, which in humans include the neurokinin-2 receptor (NK2R) and neurokinin-3 receptor (NK3R), which are encoded by TACR2 and TACR3 genes, respectively. The different neurokinin receptors are not only expressed in different areas of the body, they also have different affinities to their respective ligand of the tachykinin family. Importanly, the natural ligand of the NK1R seems to be substance $\mathrm{P}(\mathrm{SP})$, a critical neurotransmitter. Independent of the affinities to their different respective ligands, all neurokinin receptors belong to the G-protein coupled receptors (GPCRs), and several groups of $\mathrm{G}$ proteins have been found to couple to the receptor, including Gaq, Gas and the Gai $(16,17)$. Together these receptors possess a regulatory role in a broad variety of physiological and biological processes that range from the regulation of endocrine and exocrine secretions to neurological activity and here especially nociception. More recently, the neurokinin receptors have been found to have profound implications not only in the regulation of physiological cell growth but especially in inflammation and carcinogenesis. It was this latter understanding discovered in recent years which has put this receptor family, initially heavily researched back in the 1970s, into the spotlight of current anticancer research.

The NK1R has been described to have a crucial role in the carcinogenesis of a large variety of adult and childhood cancers, and several key biological and molecular concepts have become apparent. Like other GPCRs, after binding of its ligand and receptor activation, the NK1R undergoes rapid desensitization and internalization through phosphorylation processes (18). This mechanism seems to rely in part on $\beta$ arrestin/clathrin proteins and seems to be crucial for this receptor system's innate inhibitory feedback loop. Importantly, a splice variant of the NK1R was recently described that seems to have important implications in carcinogenesis by breaking up the receptor's innate feedback loop $(19,20)$.

In the event of failure to remove the intron between exons 4 and 5 during the splicing process of TACR 1 , a premature stop codon appears early, which cuts the expressed protein short. The resulting protein lacks 96 amino acids at the Cterminus and has been found to be associated with a loss of internalization and consequently its innate feedback loop. Initially, very little interest was given to the role of this truncated (tr-) splice variant of the NK1R, partially because its role was poorly understood. In an important study regarding this subject Patel et al. showed in 2005 that the stable transfection of truncated but not full length NK1R expression vectors in non-tumorigenic breast cells caused a significantly increased proliferation rate and a transformation in phenotype, indicating that in breast cancer it may be the truncated rather than the full length spice variant that is the culprit of NK1R's role in carcinogenesis (20).

What seems to be the case for breast cancer appears to be true equally for other tumors. Gillespie et al. found that it 
was the expression of tr-NK1R and not fl-NK1R that predicted the progression from quiescent colitis to high-grade dysplasia and cancer in colitis-associated cancer (21). Our own evidence published previously on this topic identified the truncated splice variant of the NK1R to be the dominant splice product in hepatoblastoma (22). In this same special issue of Anticancer Research, we further published a study analyzing primary tumor cells from children with neuroblastoma and were able to confirm that for this tumor, similarly to hepatoblastoma and other tumors, it is the truncated version of the NK1R that is highly expressed.

The NK1R system as a potent anticancer target in neuroblastoma. The NK1R has become an interesting target in a large variety of childhood cancers, including several forms of leukemia, osteosarcoma, and hepatoblastoma (2225). In a recent study Henssen et al. added neuroblastoma to this list (26). These authors demonstrated that human neuroblastoma cell lines such as IMR5, SK-N-BE, SK-NAS, SY5Y and Kelly express NK1R and can be growth inhibited both in vitro and in vivo by NK1R inhibitors. These findings are in accordings with unpublished data in the neuroblastoma cell line Kelly (Muñoz, Berger et al., unpublished observation). Henssen et al. further showed that TACR1 inhibition with NK1R inhibitors repressed E2F2 and induced TP53 signaling. Consequently, in their study, mice harboring established neuroblastoma xenograft tumors treated with aprepitant, a small molecule that inhibits NK1R activation, showed a signicantly reduced tumor burden.

The authors further found that inhibition of NK1R reduced cell viability mostly in cells with high expression levels of NK1R and its downstream target phosphorylated SRC kinase (p-SRC). Consequently, the authors observed a more pronounced induction of cell death/apoptosis in cells with high expression levels of NK1R and p-SRC. On the other hand, cells with low expression levels of NK1R and p-SRC were found to undergo cell cycle arrest rather than cell death or apoptosis. Hence, the level of expression of NK1R predicted the therapeutic response to NK1R antagonists. This effect was partially reversible by adding NK1R agonists such as SP. This latter finding identified the targeting effect of the used NK1R inhibitor to be specific (SP's is known to have a stronger binding affinity than most NK1R inhibitors, including aprepitant) $(19,27)$. These data are in accordance with our own findings recently published for hepatoblastoma and osteosarcoma $(22,23)$. Based on these data it is reasonable to believe that NK1R has a mitogenic role in neuroblastoma and establishes the NK1R as a novel therapeutic target for neuroblastoma.

Potential agents for targeting NK1R. NK1R antagonists are approved for clinical use by the Food and Drug Administration (FDA) in the form of the compound aprepitant $\left(\right.$ Emend $^{\circledR}$ ) as an antiemetic medication since 2003 . Aprepitant is a small molecule with a molecular mass of $534,43 \mathrm{~g} / \mathrm{mol}$. From a clinical perspective, aprepitant was first described to have a particularily strong effect in nausea and vomiting that is associated to chemotherapy (28). There have been several large prospective randomized clinical trials investigating the role of NK1R antagonists as antidepressants, with mixed results (29). There is strong evidence accumulating recently from a large number of prospective randomized controlled clinical trials showing efficiency of NK1R inhibitors in postoperative nausea and vomiting in both adults and children for a large variety of different surgeries (30-36). No NK1R antagonist to date is approved specifically for cancer therapy, and there are currently no ongoing clinical trials that assess their therapeutic potential regarding cancer.

When using NK1R antagonist for cancer therapy, one must consider that the dose needed to cause an anticancer effect may be several times higher compared to doses needed to create an antiemetic effect $(37,38)$. However, even high doses of NK1R antagonists have been given to humans under standardized and controlled conditions and have been found to be tolerated well (29). Importantly, while aprepitant is applied strictly orally, its prodrug fosaprepitant can be safely applied intravenously. This option can have distinct clinical advantages particularily when cancer is to be treated in children or in very advanced stages in which the gastrointestinal tract may be compromised, which is often found in advanced stages of neuroblastoma with its intensive and often debilitating therapy.

Interestingly, NK1R antagonists such as aprepitant have been successfully used as off label drugs in chronic pain. Together with nausea and vomiting as well as depression and axiety, chronic pain is one common side effect of the invasive anticancer therapy in advanced neuroblastoma. It will be interesting to investigate wheather an anticancer strategy against neuroblastoma that includes NK1R antagonists as targeted therapy can simultaneously abrogate some of these debilitating clinical side effects.

Another way of targeting the NK1R system is by targeting the NK1R's natural ligand SP, hence indirectly targeting the NK1R. This concept has been described successful in experimental settings in breast cancer and other malignant cells by using specific antibodies against SP (39, 40). Targeting SP with specific antibodies showed decreased cell survival and increased apoptosis similarly to what has been observed for direct NK1R antagonists $(39,40)$.

Future research. Many questions regarding the exact role of the $\mathrm{NK} 1 / \mathrm{SP}$ receptor system in neuroblastoma remain unanswered. Unfortunately, many previous studies - both in neuroblastoma as well as in other tumors - did not include the distinction between the full length and the truncated version of the NK1R, 
now considered a crucial component of NK1R targeting related research. In this same sense, one may ask whether the expression of the NK1R receptor, or that of its different splice variants, correlates with the clinical behavior of the tumor and could potentially predict outcome or treatment response. Although somewhat equivocal, evidence from research in breast cancer had previously suggested the existence of such a correlation (41). Our results published in this same special edition of Anticancer Research in primary neuroblastoma cells, in this sense, showed increased gene expression levels of tr$T A C R l$ and low gene expression levels of fl-TACRl. However, gene expression levels were independent from clinical markers like INSS stage, MYCN status, histology, and outcome. These results are in accordance with results we have previously published for hepatoblastoma (42).

Another important focus of future research for targeting NK1R in neuroblastoma is its downstream mechanism. It has long been described that activation of NK1R leads to phosphoinositide hydrolysis, calcium mobilization and subsequent calcium-dependent signaling activation via kinases such as SRC, at least under physiologic circumstances. Whether these same signaling mechanisms occur in cancer cells is unclear. Henssen et al. reported that NK1R inhibition in neuroblastoma repressed E2F2 and induced TP53 signaling, and that this effect may be in part due to activation of SRC (26). In a recent publication regarding the downstream signaling following NK1R inhibition in hepatoblastoma, we found potent inhibition of the AKT/mTor pathway, with subsequent strong inhibition of 4E-BP1/2 and p70S6K, both downstream members of the AKT pathway (25).

The inhibition of $\mathrm{p} 70 \mathrm{~S} 6 \mathrm{~K}$ and $4 \mathrm{E}-\mathrm{BP} 1 / 2$ created by targeting the NK1R is in accordance with other reports in breast, colon and prostate cancer cells (39). In addition to the inhibition of the AKT pathway, in hepatoblastoma cells we recently described a flagrant reduction of FOXM1. FOXM1 is a key interacting protein of the canonical Wnt pathway and here responsible for the translocation of $\beta$-catenin into the nucleus. This potent crosslink between the NK1R and the canonical Wnt pathway, until then believed to be two completely separate tumor targets, was the first time an inhibition of the Wnt pathway has been described by targeting the SP/NK1R complex (25). Certainly, this new insight opens hope for therapeutic options in which both NK1R and the canonical Wnt pathway could be targeted simultaneously to generate a strong anticancer effect. Future research will show whether such a potent crosslink exists for NK1R downstream signaling in neuroblastoma.

One other interesting field of ongoing research in neuroblastoma is the role of cancer stem cells (CSCs). CSCs represent a small fraction of tumor cells within a given tumor that are similar to but hierarchically superior to regular tumor cells. These cells typically demonstrate properties that are similar to physiologic stem cells needed for tissue renewal and healing under normal conditions. In tumors, compared to normal cells they have been found to have a capacity for self-renewal, proliferate more viciously, and are heavily responsible for tumor maintenance and metastasis. Importantly, CSCs have been found to be exponentially more resistant to chemotherapy and radiation compared to their normal tumor cell counterparts. Consequently, CSCs are currently being heavily researched in order to generate stronger, more effective and more lasting anticancer tools.

Although somewhat tricky to characterize, CSCs have successfully been described in neuroblastoma (43-45). Typical cell surface markers that have been associated with CSCs in neuroblastoma are CD114, CD117, CD133, and ABCG2 (44). There is now accumulating evidence for hepatoblastoma that NK1R may be highly expressed in CSCs of that tumor and can be readily targeted with NK1R antagonists. For example, our data show that the application of NK1R antagonists not only diminished the sphere-formation capacity of hepatoblastoma cells, but also decreased the expression of liver-specific CSC markers and selected ESC genes (25). Importantly, liver specific CSCs could be successfully targeted with NK1R antagonists such as aprepitant. Whether such potential exists for neuroblastoma remains to be clarified but would be an interesting approach for further investigation in the field of neuroblastoma research.

\section{Conclusion}

Despite significant advances in the field of neuroblastoma research high-risk neuroblastoma continues to have a poor prognosis. The NK1R system has been described to have a significant role in the carcinogenesis of a large variety of tumors, including many childhood cancers. Consequently, NK1R is a promising anticancer target and NK1R inhibitors such as the clinical drugs aprepitant and its prodrug fosaprepitant are considered potent and promising anticancer agents. Although research on the NK1/SP receptor system is limited in neuroblastoma, it appears from new evidence that NK1R is expressed in neuroblastoma independenly of its subtype and that NK1R inhibitors may be a new and promising target in childhood neuroblastoma. More research is needed to clarify the exact role of the NK1/SP receptor system in neuroblastoma in order to elucidate its exact biological role and its clinical implications.

\section{Conflicts of Interest}

The Authors declare that they have no conflicts of interest.

\section{References}

1 Irwin MS and Park JR: Neuroblastoma: paradigm for precision medicine. Pediatr Clin North Am 62: 225-256, 2015. 
2 Davidoff AM: Neuroblastoma. Seminars in Pediatric Surgery 21: 2-14, 2012.

3 Kaneko Y, Kanda N, Maseki N, Tsuchida Y, Takeda T, Okabe I and Sakurai M: Different karyotypic patterns in early and advanced stage neuroblastomas. Cancer Research 47: 311-318, 1987.

4 London WB, Castleberry RP, Matthay KK, Look AT, Seeger RC, Shimada H, Thorner P, Brodeur G, Maris JM, Reynolds CP and Cohn SL: Evidence for an age cutoff greater than 365 days for neuroblastoma risk group stratification in the Children's Oncology Group. J Clin Oncol 23: 6459-6465, 2005.

5 Moroz V, Machin D, Faldum A, Hero B, Iehara T, Mosseri V, Ladenstein R, De Bernardi B, Rubie H, Berthold F, Matthay KK, Monclair T, Ambros PF, Pearson ADJ, Cohn SL and London WB: Changes over three decades in outcome and the prognostic influence of age-at-diagnosis in young patients with neuroblastoma: a report from the International Neuroblastoma Risk Group Project. Eur J Cancer 47: 561-571, 2011.

6 Monclair T, Brodeur GM, Ambros PF, Brisse HJ, Cecchetto G, Holmes K, Kaneko M, London WB, Matthay KK, Nuchtern JG, von Schweinitz D, Simon T, Cohn SL, Pearson ADJINRG Task Force: The International Neuroblastoma Risk Group (INRG) staging system: an INRG Task Force report. J Clin Oncol 27: 298-303, 2009.

7 Chatten J, Shimada H, Sather HN, Wong KY, Siegel SE and Hammond GD: Prognostic value of histopathology in advanced neuroblastoma: a report from the Childrens Cancer Study Group. Hum Pathol 19: 1187-1198, 1988.

8 Seeger RC, Brodeur GM, Sather H, Dalton A, Siegel SE, Wong $\mathrm{KY}$ and Hammond D: Association of multiple copies of the Nmyc oncogene with rapid progression of neuroblastomas. N Engl J Med 313: 1111-1116, 1985.

9 Brodeur GM, Seeger RC, Schwab M, Varmus HE and Bishop JM: Amplification of N-myc in untreated human neuroblastomas correlates with advanced disease stage. Science 224: 1121-1124, 1984.

10 Esposito MR, Aveic S, Seydel A and Tonini GP: Neuroblastoma treatment in the post-genomic era. J Biomed Sci 24: 14, 2017.

11 Cheung NK, Burch L, Kushner BH and Munn DH: Monoclonal antibody 3F8 can effect durable remissions in neuroblastoma patients refractory to chemotherapy: a phase II trial. Prog Clin Biol Res 366: 395-400, 1991.

12 Hank JA, Surfus J, Gan J, Chew TL, Hong R, Tans K, Reisfeld $\mathrm{R}$, Seeger RC, Reynolds $\mathrm{CP}$ and Bauer M: Treatment of neuroblastoma patients with antiganglioside GD2 antibody plus interleukin-2 induces antibody-dependent cellular cytotoxicity against neuroblastoma detected in vitro. J Immunother Emphasis Tumor Immunol 15: 29-37, 1994.

13 Wood AC, Krytska K, Ryles HT, Infarinato NR, Sano R, Hansel TD, Hart LS, King FJ, Smith TR, Ainscow E, Grandinetti KB, Tuntland T, Kim S, Caponigro G, He YQ, Krupa S, Li N, Harris JL and Mossé YP: Dual ALK and CDK4/6 Inhibition Demonstrates Synergy against Neuroblastoma. Clin Cancer Res 23: 2856-2868, 2017

14 Bresler SC, Weiser DA, Huwe PJ, Park JH, Krytska K, Ryles H, Laudenslager M, Rappaport EF, Wood AC, McGrady PW, Hogarty MD, London WB, Radhakrishnan R, Lemmon MA and Mossé YP: ALK mutations confer differential oncogenic activation and sensitivity to ALK inhibition therapy in neuroblastoma. Cancer Cell 26: 682-694, 2014.
15 Mossé YP, Deyell RJ, Berthold F, Nagakawara A, Ambros PF, Monclair T, Cohn SL, Pearson AD, London WB and Matthay KK: Neuroblastoma in older children, adolescents and young adults: a report from the International Neuroblastoma Risk Group project. Pediatr Blood Cancer 61: 627-635, 2014.

16 Mitsuhashi M, Ohashi Y, Shichijo S, Christian C, SudduthKlinger J, Harrowe G and Payan DG: Multiple intracellular signaling pathways of the neuropeptide substance $\mathrm{P}$ receptor. $\mathrm{J}$ Neurosci Res 32: 437-443, 1992.

17 Roush ED and Kwatra MM: Human substance P receptor expressed in Chinese hamster ovary cells directly activates G(alpha q/11), G(alpha s), G(alpha o). FEBS Lett 428: 291-294, 1998.

18 Mantyh PW, Allen CJ, Ghilardi JR, Rogers SD, Mantyh CR, Liu $\mathrm{H}$, Basbaum AI, Vigna SR and Maggio JE: Rapid endocytosis of a $G$ protein-coupled receptor: substance $P$ evoked internalization of its receptor in the rat striatum in vivo. Proc Natl Acad Sci USA 92: 2622-2626, 1995.

19 Lindström E, Mentzer von B, Påhlman I, Ahlstedt I, Uvebrant A, Kristensson E, Martinsson R, Novén A, de Verdier J and Vauquelin G: Neurokinin 1 receptor antagonists: correlation between in vitro receptor interaction and in vivo efficacy. J Pharmacol Exp Ther 322: 1286-1293, 2007.

20 Patel HJ, Ramkissoon SH, Patel PS and Rameshwar P: Transformation of breast cells by truncated neurokinin-1 receptor is secondary to activation by preprotachykinin-A peptides. Proc Natl Acad Sci USA 102: 17436-17441, 2005.

21 Gillespie E, Leeman SE, Watts LA, Coukos JA, O’Brien MJ, Cerda SR, Farraye FA, Stucchi AF and Becker JM: Truncated neurokinin-1 receptor is increased in colonic epithelial cells from patients with colitis-associated cancer. Proceedings of the National Academy of Sciences 108: 17420-17425, 2011.

22 Berger M, Neth O, Ilmer M, Garnier A, Salinas-Martín MV, de Agustín Asencio JC, von Schweinitz D, Kappler R and Muñoz M: Hepatoblastoma cells express truncated neurokinin-1 receptor and can be growth inhibited by aprepitant in vitro and in vivo. J Hepatol 60: 985-994, 2014.

23 Muñoz M, Berger M, Rosso M, Gonzalez-Ortega A, Carranza A and Coveñas R: Antitumor activity of neurokinin-1 receptor antagonists in MG-63 human osteosarcoma xenografts. Int J Oncol 44: 137-146, 2014.

24 Garnier A, Vykoukal J, Hubertus J, Alt E, von Schweinitz D, Kappler R, Berger M and Ilmer M: Targeting the neurokinin-1 receptor inhibits growth of human colon cancer cells. Int J Oncol 47: 151-160, 2015.

25 Ilmer M, Garnier A, Vykoukal J, Alt E, von Schweinitz D, Kappler R and Berger M: Targeting the Neurokinin-1 receptor compromises canonical Wnt signaling in hepatoblastoma. Mol Cancer Ther 14: 2712-2721, 2015.

26 Henssen AG, Odersky A, Szymansky A, Seiler M, Althoff K, Beckers A, Speleman F, Schäfers S, De Preter K, Astrahanseff K, Struck J, Schramm A, Eggert A, Bergmann A and Schulte JH: Targeting tachykinin receptors in neuroblastoma. Oncotarget 8 : 430-443, 2017.

27 Rosso M, Muñoz M and Berger M: The Role of Neurokinin-1 Receptor in the Microenvironment of Inflammation and Cancer. The Scientific World Journal 2012: 1-21, 2012.

28 Aapro M, Carides A, Rapoport BL, Schmoll H-J, Zhang L and Warr D: Aprepitant and fosaprepitant: a 10-year review of efficacy and safety. Oncologist 20: 450-458, 2015. 
29 Kramer MS: Distinct Mechanism for Antidepressant Activity by Blockade of Central Substance P Receptors. Science 281: 1640$1645,1998$.

30 Liu M, Zhang H, Du B-X, Xu F-Y, Zou Z, Sui B and Shi X-Y: Neurokinin-1 receptor antagonists in preventing postoperative nausea and vomiting: a systematic review and meta-analysis. Medicine (Baltimore) 94: e762, 2015.

31 Jacob D, Busciglio IA, Burton DD, Halawi H, Oduyebo I, Rhoten D, Ryks M, Harmsen WS and Camilleri M: Effects of NK1 receptors on gastric motor functions and satiation in healthy humans: Results from a controlled trial with the NK1 antagonist, aprepitant. Am J Physiol Gastrointest Liver Physiol, 2017. doi: 10.1152/ajpgi.00197.2017. [Epub ahead of print]

32 Therneau IW, Martin EE, Sprung J, Kellogg TA, Schroeder DR and Weingarten TN: The Role of Aprepitant in Prevention of Postoperative Nausea and Vomiting After Bariatric Surgery. Obes Surg 347: 498, 2017.

33 Ham SY, Shim YH, Kim EH, Son MJ, Park WS and Lee JS: Aprepitant for antiemesis after laparoscopic gynaecological surgery: A randomised controlled trial. Eur J Anaesthesiol 33: 90-95, 2016.

34 Singh PM, Borle A, Rewari V, Makkar JK, Trikha A, Sinha AC and Goudra B: Aprepitant for postoperative nausea and vomiting: a systematic review and meta-analysis. Postgrad Med J 92: 87-98, 2016.

35 Milnes V, Gonzalez A and Amos V: Aprepitant: A New Modality for the Prevention of Postoperative Nausea and Vomiting: An Evidence-Based Review. J Perianesth Nurs 30: 406-417, 2015.

36 Trimas SJ and Trimas MD: Use of aprepitant and factors associated with incidence of postoperative nausea and vomiting in patients undergoing facial plastic surgery. JAMA Facial Plast Surg 17: 251-255, 2015.

37 Muñoz $M$ and Rosso $M$ : The NK-1 receptor antagonist aprepitant as a broad spectrum antitumor drug. Invest New Drugs 28: 187-193, 2010.

38 Muñoz M, Rosso M and Coveñas R: A new frontier in the treatment of cancer: NK-1 receptor antagonists. Curr Med Chem 17: 504-516, 2010
39 Mayordomo C, García-Recio S, Ametller E, Fernández-Nogueira P, Pastor-Arroyo EM, Vinyals L, Casas I, Gascón P and Almendro V: Targeting of substance $P$ induces cancer cell death and decreases the steady state of EGFR and Her2. J Cell Physiol 227: 1358-1366, 2012.

40 Garcia-Recio S, Fuster G, Fernandez-Nogueira P, Pastor-Arroyo EM, Park SY, Mayordomo C, Ametller E, Mancino M, Gonzalez-Farre X, Russnes HG, Engel P, Costamagna D, Fernandez PL, Gascon P and Almendro V: Substance P autocrine signaling contributes to persistent HER2 activation that drives malignant progression and drug resistance in breast cancer. Cancer Research 73: 6424-6434, 2013.

41 Zhou Y, Zhao L, Xiong T, Chen X, Zhang Y, Yu M, Yang J and Yao Z: Roles of full-length and truncated neurokinin-1 receptors on tumor progression and distant metastasis in human breast cancer. Breast Cancer Res Treat 140: 49-61, 2013.

42 Garnier A, Ilmer M, Becker K, Häberle B, von Schweinitz D, Kappler R and Berger M: Truncated neurokinin-1 receptor is an ubiquitous antitumor target in hepatoblastoma, and its expression is independent of tumor biology and stage. Oncol Lett 11: 870$878,2016$.

43 Hansford LM, McKee AE, Zhang L, George RE, Gerstle JT, Thorner PS, Smith KM, Look AT, Yeger H, Miller FD, Irwin MS, Thiele CJ and Kaplan DR: Neuroblastoma cells isolated from bone marrow metastases contain a naturally enriched tumor-initiating cell. Cancer Research 67: 11234-11243, 2007.

44 Garner EF and Beierle EA: Cancer Stem Cells and Their Interaction with the Tumor Microenvironment in Neuroblastoma. Cancers (Basel) 8: 5, 2015.

45 Walton JD, Kattan DR, Thomas SK, Spengler BA, Guo H-F, Biedler JL, Cheung N-KV and Ross RA: Characteristics of stem cells from human neuroblastoma cell lines and in tumors. Neoplasia 6: 838-845, 2004.

Received August 19, 2017

Revised September 10, 2017 Accepted September 11, 2017 